\title{
FEATURED ARTICLES
}

Bace-ic instinct

Neuroscience Gateway (December 2006) | doi:10.1038/aba1703

The protease Bace1, which is associated with Alzheimer disease, is necessary for the proper formation of myelin sheaths.

According to the novel Wicked, the Wicked Witch of the West from the Wizard of $\mathrm{Oz}$ was actually a maligned, but well-meaning political activist. Similarly, many disease-associated proteins are important in benign cellular functions. Bace 1 is one of the enzymes that convert amyloid precursor protein into the amyloid protein $A \beta$, which may cause Alzheimer disease in people. Hu et al. report that Bace 1 is also important in myelination in a recent article in Nature Neuroscience.

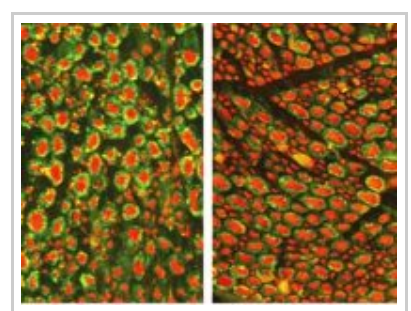

Sciatic nerves are hypomyelinated in Bace 1 knockout (right) relative to wildtype (left) mice. Myelin-specific immunostaining for MBP and axon-specific immunostaining for neurofilament are shown in green and red, respectively.

Because Bace 1 localizes to axons and associates with a neurite growth inhibitor, the authors proposed that Bace 1 would be important in axon growth. Although Bace1 knockout and wild-type mice showed similar axon development, Bace1 knockouts showed reduced expression of myelin basic protein (MBP) relative to wildtype mice, suggesting that Bace1 knockouts had less myelin than did wild-type mice. The g-ratio is a measure of myelination independent of axon diameter. Bace1-null optic and sciatic nerves had reduced $\mathrm{g}$-ratios relative to wild-type optic and sciatic nerves, suggesting that relative to wild-type mice, Bace1 knockout mice showed reduced myelination in both the central and peripheral nervous systems. In mice with myelination defects, the majority of myelinated axons have small diameters. Approximately $83 \%$ of myelinated axons in Bace 1 knockouts and roughly $66 \%$ of myelinated axons in wild-type mice had diameters less than $3 \mu \mathrm{m}$.

In the absence of Bace 1, myelination was not completely impaired. Because Bace1 knockout axons had thin myelin sheaths, the authors predicted deficits in compact myelin, the multilayered stack of membrane sheets, but not in periaxonal membrane, the membrane of myelin closest to the axon. Relative to wild-type mice, Bace1 knockouts showed reductions in MBP and proteolipid protein, which localize to compact myelin, but had similar levels of myelin-associated glycoprotein, which localizes to the periaxonal membrane.

Demyelinating diseases can cause chronic pain and weakness. Bace1 knockouts withdrew their paws from a hot-plate faster than did wild-type mice, suggesting that they had increased sensitivity to pain. Bace1 knockouts also had weaker grips relative to wildtype mice. Therefore, Bace 1 deficiency caused neurological symptoms consistent with myelin pathology.

Neuregulin-1 regulates the thickness of myelin sheaths. Bace 1 knockout brains had more full-length neuregulin-1 and less of its $\mathrm{N}$-terminal fragment than did wild-type brains, suggesting that Bace 1 digests neuregulin-1 into smaller fragments. Neuregulin-1 
binds ErbB receptors and activates the serine/threonine kinase Akt. Although Bace1 knockout and wild-type brains had similar amounts of Akt, Bace1 knockout brains showed reduced phosphorylation of Akt relative to wild-type brains. Akt activation regulates the expression of MBP and proteolipid protein.

Therefore, the authors conclude that Bace1-digested neuregulin-1 activates Akt signaling at ErbB receptors to regulate the expression of myelin proteins.

Bace1 inhibitors may limit disease progression in people with Alzheimer disease. However, if in addition to myelin formation, neuregulin is necessary for the maintenance of myelin, then Bace1 inhibition may cause more problems than it solves.

\section{Debra Speert}

1. Hu, X. et al.Bace 1 modulates myelination in the central and peripheral nervous system. Nature Neuroscience 9, 15201525 (2006). | Article | 